\title{
Mesenchymal stem cells for Glenoid chondral lesions: An exceptional use
}

Vanessa Valim ${ }^{1}$, Annelise Pezzi ${ }^{1}$, Bruna Amorin ${ }^{1,2}$, Bruna Zambonato ${ }^{1}$, Álvaro Laureano ${ }^{1}$, Maria Aparecida Lima da Silva ${ }^{1}$, Alice Dahmer ${ }^{1}$ Filipe Sehn', João L Ellera Gomes ${ }^{3}$ and Lucia Silla ${ }^{1,3 *}$

${ }^{1}$ Cell Therapy and Technology Center, Hospital de Clinicas de Porto Alegre, HCPA, Porto Alegre, Brazil

${ }^{2}$ Centro Universitário Ritter dos Reis - UniRitter Porto Alegre, Brazil

${ }^{3}$ School of Medicine, Universidade Federal do Rio Grande do Sul, UFRGS, Porto Alegre, Brazil

\begin{abstract}
Background: Damage to the articular cartilage is usually caused by accidental or athletic injuries or trauma often progressing to more severe joint diseases, such as osteoarthritis, subchondral bone necrosis, and arthritis. It is estimated that $15 \%$ of the world's adult population is affected by joint diseases. The long-term success of cartilage repair depends on the development of alternative regenerative approaches. In recent years, the use of stem cells for the treatment of degenerative diseases has been widely explored. Here we report a case of exceptional use of autologous bone marrow mesenchymal stem cells expanded in medium supplemented with autologous platelet lysate in a patient with glenoid chondral erosion.
\end{abstract}

Methods: A 17-year-old patient was diagnosed as having chondral erosion of the center of the labrum in the right shoulder, with symptoms of pain and insecurity in the right upper limb. Thirty days before arthroscopy, autologous platelets and stem cells were obtaind from apheresis and BM aspiration, respectively. Arthroscopy was then performed with the patient in the lateral decubitus position with arm abducted to $45^{\circ}$ under manual traction. Microfracture was performed in the central region defect, and MSC intra-articular injection was performe after extensive washing with sterile saline.

Discussion: No adverse reactions were observed. Two years after arthroscopy, there were no sign of lesion in cartilage, rotator cuff or biceps tendon were observed in magnetic resonance imaging. Further studies are needed to validate the clinical efficacy and safety of MSC injection for glenoid chondral defect treatment.

Trial registration: The procedure was approved by Hospital de Clínicas de Porto Alegre Research Ethics Committee - protocol number 14-0545.

Abbreviations: MSC - mesenchymal stem cells; aPL - platelet lysate; HCPA - Hospital de Clínicas de Porto Alegre; MRI - Magnetic resonance imaging.

\section{Background}

Damage to the articular cartilage is usually caused by accidental or athletic injuries or trauma, often progressing to more severe joint diseases, such as osteoarthritis, subchondral bone necrosis, and arthritis. After traumatic or pathological hyaline articular cartilage damage, the load-bearing joint surface has little or no intrinsic ability to repair even minor damage, which may lead to progressive damage and joint degeneration $[1,2]$.

It is estimated that $15 \%$ of the world's adult population is affected by joint diseases. Current treatments for articular cartilage damage, including surgery (microfracture and implantation of autologous chondrocytes or allografts), seek to repair cartilage defects, but they are rarely able to fully restore the joint function. In fact, in the long term, these techniques may promote the development of fibrocartilage rather than the formation of the desired hyaline cartilage [3]. Therefore, cartilage repair and presence of long-term damage remain a challenge in orthopedic surgery.

The long-term success of cartilage repair depends on the development of alternative regenerative approaches [4]. In recent years, the use of stem cells for the treatment of degenerative diseases has been widely explored. Studies have been conducted in the fields of cardiology, neurology, and dentistry. However, investigations in the orthopedic field are still scarce [3]. Given the difficulty in surgically repairing cartilage lesions, it was hypothesized that injection of mesenchymal stem cells (MSCs) into connective tissue, such as tendon and cartilage, would stimulate repair, as observed in other tissues [5].

MSCs are multipotent adult stem cells that can differentiate into chondrogenic cells. Thus, a series of strategic experiments has been designed to investigate whether MSCs are good candidates for the maintenance and regeneration of articular cartilage [6]. Another reason for the use of MSCs is their trophic and regenerative potential, which may be valuable in the treatment of chronic degenerative diseases, thereby preventing cartilage degradation in the affected joints.

In 2010, the first clinical study to evaluate the use of autologous MSCs supplemented with human platelet lysate for the treatment of articular cartilage defects in humans was published. Patients were followed for 12 months after the implantation procedure and all of them showed an improvement in symptoms at 12 months [7]. In a study

Correspondence to: Lucia Silla, Cell Therapy and Technology Center, Hospital de Clinicas de Porto Alegre, HCPA, Porto Alegre, Ramiro Barcelos 2350-90035903, Brazil, Tel: 55513359 8000; E-mail: 1silla@hcpa.edu.br

Key words: Mesenchymal stem cell, glenoid chondral, lesion

Received: November 06, 2017; Accepted: November 27, 2017; Published: November 29, 2017 
conducted in 2012, the implantation of autologous mononuclear bone marrow cells in rotator cuff sutures appeared to be an alternative to other biological approaches for the maintenance of hard tissue integrity, thereby decreasing the occurrence of retears [8].

We report a case of exceptional use of autologous bone marrow MSCs expanded in medium supplemented with autologous platelet lysate (aPL) in a patient with glenoid chondral erosion. The patient was monitored at the Orthopedic Trauma Service of Hospital de Clínicas de Porto Alegre (HCPA) and offered the option of undergoing this procedure as an alternative treatment for glenoid chondral lesions in the shoulder in the absence of other effective treatment options. The procedure was approved by HCPA Research Ethics Committee (protocol number 14-0545), and written informed consent was obtained from the patient prior to initiation of treatment. Written permission was also obtained from the patient to publish this study protocol.

\section{Methods}

\section{Case report}

A 17-year-old male patient was diagnosed as having chondral erosion of the center of the labrum in the right shoulder, with symptoms of pain and insecurity in the right upper limb. Magnetic resonance imaging (MRI) revealed an osteochondral lesion on the posteriorsuperior glenoid rim measuring about $1.5 \times 1.3 \mathrm{~cm}$, with the presence of an area of signal intensity similar to that of the fluid surrounding the lesion, a finding that suggests instability (Figure 1 ). The patient received medical treatment and underwent physical therapy without satisfactory results.

Arthroscopy was then performed with the patient in the lateral decubitus position with the right arm abducted to $45^{\circ}$ under manual traction. The joint activity was examined and a grade III/IV cartilage defect measuring $1.5 \mathrm{~cm}$ in diameter was identified in the central portion of the glenoid cavity. Debridement was performed with removal of loose bodies. Microfracture was performed with emphasis on the central region of the defect. The joint was washed with sterile saline and continuous drainage was performed using the two portals of the shoulder joint, with subsequent injection of MSCs.

\section{MSC preparation}

MSCs were derived from bone marrow aspirates obtained from the patient 30 days before the arthroscopic procedure. A total of 120 $\mathrm{mL}$ of bone marrow was collected and mononuclear cells were isolated by density gradient centrifugation using Ficoll Histopaque (Sigma-

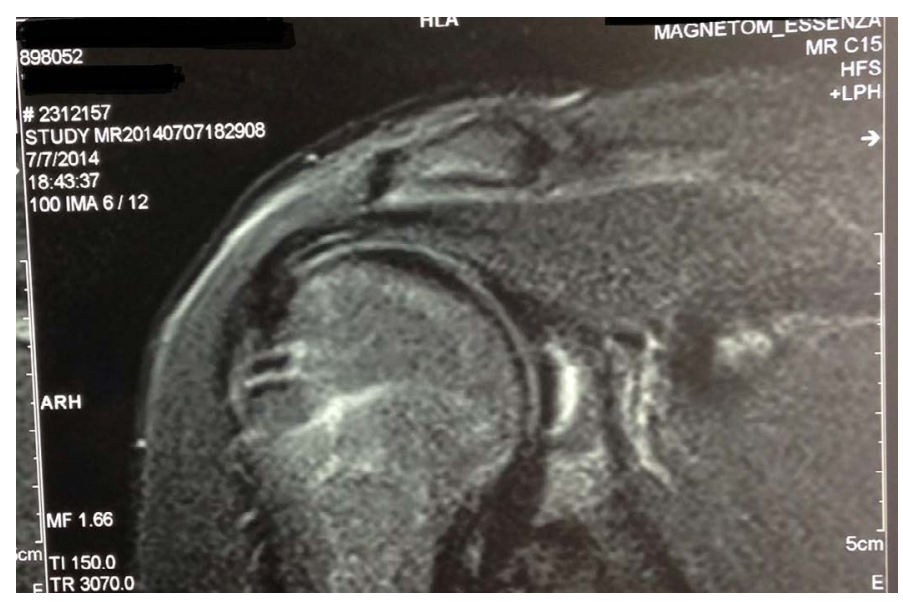

Figure 1. MRI showing an osteochondral lesion in the glenoid cavity.
Aldrich). The isolated cells were cultured in low-glucose DMEM (Gibco) supplemented with $10 \%$ aPL $+1 \%$ penicillin/streptomycin (Gibco) in order to isolate and expand MSCs. The cells were cryopreserved at the second passage for subsequent quality control testing (immunophenotyping, microbiological assay, and mycoplasma and endotoxin detection assays). After testing, the samples were ready for use. On the day of injection, the cells were thawed in a $37^{\circ} \mathrm{C}$ water bath, washed to remove DMSO and resuspended in $10 \mathrm{~mL}$ of aPL in a syringe. During the procedure, $1.4 \times 10^{7}$ viable MSCs were injected.

\section{aPL preparation}

For aPL preparation, an apheresis was performed in the patient and aPL was produced according to good manufacturing practices before cell culture. The same aPL was used as a suspension medium for cell injection. After quality control testing (endotoxin detection and microbiological assays), aPL was ready for use.

\section{Assessment of treatment response}

Immediately after surgery, no adverse reactions were observed with the injection of MSCs.

Two years after arthroscopy (right shoulder), MRI revealed no signs of damage on the rotator cuff tendon or biceps tendon. The attachment of the labrum and biceps tendon to the glenoid was preserved, with a normal muscle belly. The articular cartilage of the glenohumeral joint was preserved and there was no significant joint effusion (Figure 2).

\section{Discussion}

To date, MSCs have been used in a few cases of articular cartilage damage, with satisfactory results in some cases of rotator cuff injury $(8,9)$. The present study is the first to report the exceptional use of autologous MSCs in a case of glenoid chondral defect. Despite the satisfactory results, further studies are needed to validate the clinical efficacy and safety of this MSC injection protocol for cases of glenoid chondral defect.

\section{Declarations}

\section{Ethics approval and consent to participate and for publication}

The procedure was approved by Hospital de Clínicas de Porto Alegre (HCPA) Research Ethics Committee (protocol number 140545), and written informed consent was obtained from the patient prior to initiation of treatment. Written permission was also obtained from the patient to publish this study protocol.

\section{Availability of data and material}

All data generated or analysed during this study are included in this published article

\section{Competing interests}

The authors have no commercial, proprietary, or financial interest in the products or companies described in this article.

\section{Funding}

Financial supports from: $\mathrm{CNPq}$, CAPES, INCT, Regenera and FINEP.

\section{Authors' contributions}

V.V - MSC culture and article writing

A.P - aPL procedure and article writing 


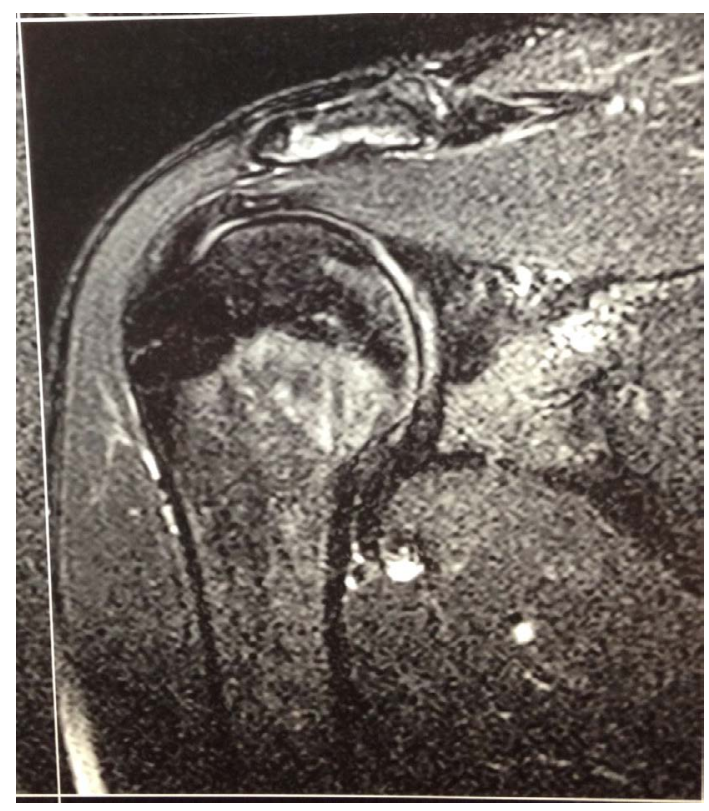

Figure 2. MRI coronal T2-weighted fat-saturated images with no evidence of damage.

B.A - MSC culture and article writing

B.Z - Follow-up of patient evolution and article revision

A.L - cell quality control and article revision

M.A.L.S - cell quality control and article revision

A.D - cell quality control and article revision

F.S - Review about the subject

J.E.G - arthroscopy and mesenchymal stem cell infusion and article revision

L.S - Head of the cell therapy center and quality assurance and article revision

\section{Acknowledgements}

Support Fund for Research and Events of Hospital de Clínicas de Porto Alegre (FIPE-HCPA), Financiadora de Estudos e Projetos (FINEP), Coordenação de Aperfeiçoamento de Pessoal de Nível Superior (CAPES) and Conselho Nacional de Desenvolvimento Científico e Tecnológico (CNPq).

\section{References}

1. Csaki C, Schneider PR, Shakibaei M (2008) Mesenchymal stem cells as a potential pool for cartilage tissue engineering. Ann Anat 190: 395-412. [Crossref]

2. Davatchi F, Abdollahi BS, Mohyeddin M, Shahram F, Nikbin B (2011) Mesenchymal stem cell therapy for knee osteoarthritis. Preliminary report of four patients. Int $J$ Rheum Dis 14: 211-215. [Crossref]

3. Wang Y, Yuan M, Guo QY, Lu SB, Peng J (2015) Mesenchymal Stem Cells for Treating Articular Cartilage Defects and Osteoarthritis. Cell Transplant 24: 1661-1678. [Crossref]

4. Brittberg M, Peterson L, Sjogren-Jansson E, Tallheden T, Lindahl A (2003) Articular cartilage engineering with autologous chondrocyte transplantation. A review of recent developments. J Bone Joint Surg Am 85-A Suppl 3: 109-115.

5. Connelly JT, Wilson CG, Levenston ME (2008) Characterization of proteoglycan production and processing by chondrocytes and BMSCs in tissue engineered constructs. Osteoarthritis Cartilage 16: 1092-1100.

6. Berninger MT, Wexel G, Rummeny EJ, Imhoff AB, Anton M, et al. (2013) Treatment of osteochondral defects in the rabbit's knee joint by implantation of allogeneic mesenchymal stem cells in fibrin clots. J Vis Exp: e4423. [Crossref]

7. Haleem AM, Singergy AA, Sabry D, Atta HM, Rashed LA, et al. (2010) The Clinical Use of Human Culture-Expanded Autologous Bone Marrow Mesenchymal Stem Cells Transplanted on Platelet-Rich Fibrin Glue in the Treatment of Articular Cartilage Defects: A Pilot Study and Preliminary Results. Cartilage 1: 253-261.

8. Ellera Gomes JL, da Silva RC, Silla LM, Abreu MR, Pellanda R (2012) Conventional rotator cuff repair complemented by the aid of mononuclear autologous stem cells. Knee Surg Sports Traumatol Arthrosc 20: 373-377.

9. Hernigou P, Flouzat Lachaniette CH, Delambre J, Zilber S, Duffiet P, et al. (2014) Biologic augmentation of rotator cuff repair with mesenchymal stem cells during arthroscopy improves healing and prevents further tears: a case-controlled study. Int Orthop 38: 1811-1818. [Crossref]

Copyright: (C2017 Valim V. This is an open-access article distributed under the terms of the Creative Commons Attribution License, which permits unrestricted use, distribution, and reproduction in any medium, provided the original author and source are credited. 